\title{
A Multicentre Randomized Clinical Trial on Efficacy and Safety of Huxin Formula in Patients Undergoing Percutaneous Coronary Intervention
}

\author{
Huan-Lin Wu, ${ }^{1}$ Yun-Fei Wang, ${ }^{1}$ Jun-Zhe Li, ${ }^{1}$ Min-Zhou Zhang, ${ }^{1}$ Xiao-Gang Sheng, \\ Xia Wang, ${ }^{1}$ Song Li, ${ }^{1}$ Qiu-Xiong Chen, ${ }^{1}$ Xiao-Qing Li, ${ }^{1}$ Ai-hua Ou, ${ }^{2}$ and Xin-Min Ruan ${ }^{1}$ \\ ${ }^{1}$ Heart Center, The Second Affiliated Hospital of Guangzhou University of Chinese Medicine, Guangzhou 510020, China \\ ${ }^{2}$ Department of DME, Guangzhou University of Chinese Medicine, Guangzhou 510020, China
}

Correspondence should be addressed to Xin-Min Ruan; rxmmd@163.com

Received 21 January 2014; Revised 6 May 2014; Accepted 7 May 2014; Published 26 May 2014

Academic Editor: Arthur Sá Ferreira

Copyright (C) 2014 Huan-Lin Wu et al. This is an open access article distributed under the Creative Commons Attribution License, which permits unrestricted use, distribution, and reproduction in any medium, provided the original work is properly cited.

\begin{abstract}
Percutaneous coronary intervention (PCI) is widely used in clinical treatment of coronary artery disease. However, the effects of PCI on preventing restenosis after revascularization and improving the quality of life were not satisfying. Huxin Formula is formulated by modifying an experienced Chinese medicine formula and has been widely used in clinical practice due to its marked effects on coronary heart disease. A multicentre double-blind randomized controlled clinical trial was designed to evaluate the effects and safety of Huxin Formula in patients undergoing PCI. Our results showed that there was no significant difference between the two groups in main outcomes. For patients with ejection fraction $(\mathrm{EF})>50 \%$, score of the quality of life scale was higher in treatment group compared with control group. For patients with unstable angina, score of the quality of life scale in 360 days was significantly higher in treatment group compared with control group $(P<0.05)$. No obvious adverse reaction was found in the use of Huxin Formula. In conclusion, Huxin Formula, believed to be a safe treatment for patients after PCI, has benefits in improving the quality of life in patients with unstable angina though it failed to show superiority in primary and secondary outcomes.
\end{abstract}

\section{Introduction}

Coronary heart disease causes huge damage in human health and lives. Percutaneous coronary intervention (PCI) and coronary artery bypass grafting (CABG), which greatly reduce the incidence of cardiovascular events and alleviate the symptoms, are widely used in clinical treatment since the development of modern medicine. However, the effects of both treatments on preventing restenosis after revascularization and improving the quality of life were not satisfying. Recent studies had showed that traditional Chinese medicine (TCM) has advantages in preventing restenosis after PCI and improving the quality of life, but these trials had limited internal validity due to poor design and small sample size.

Huxin Formula is formulated by modifying an experienced Chinese medicine formula which is created by a wellknown Chinese doctor, Professor DENG Tie-tao, and has been widely used in clinical practice due to its marked effects on coronary heart disease.

In this trial, we evaluated the efficacy and safety of Huxin Formula in patients after PCI using a multicenter, doubleblind, randomized, and placebo-controlled clinical trial.

\section{Methods}

2.1. Subjects. Participants were screened by investigators at 12 first-rate domestic hospitals in China, including Guangdong Provincial Hospital of Chinese Medicine, Guangdong Provincial People's Hospital, Xinjiang Hospital of Chinese Medicine, Jiangsu Provincial Hospital of Chinese Medicine, The No. 10 Hospital of Shanghai, Shanxi Provincial People's Hospital, Gansu Provincial People's Hospital, The First Affiliated Hospital of Sun Yat-sen University, The First Affiliated Hospital of Guangzhou University of TCM, Huaqiao Hospital 
TABLE 1: Inclusion and exclusion criteria.

Inclusion criteria
(1) Age 40 to 75 without gender limitation
(2) Patients who meet the diagnostic criteria of unstable angina or
myocardial infarction
(3) Patients who were diagnosed as "deficiency of heart-QI and
phlegm stasis-bizu" according to traditional Chinese medicine
(TCM)
(4) One week after PCI
Exclusion criteria
(1) Patients who have acute myocardial infarction or severe heart
failure (cardiac function class IV according to the cardiac function
standard of New York Heart Association and the left ventricular
ejection fraction $\leq 30 \%$ by heart color Doppler)
(2) Patients who have severe liver and kidney dysfunction (serum
alanine aminotransferase > three times the normal cap and/or
plasma creatinine $\geq 442$ umol/L)
(3) Patients who have cancer or active gastrointestinal bleeding
(4) Patients with mental illness
(5) Patients who were pregnant or breast-feeding
(6) Patients who have medical history of CABG and undergo PCI
in vascular bridge
(7) Patients with stable angina

of Jinan University, Jiangmen Wuyi Hospital of TCM, and People's Hospital of Liuzhou. The study was conducted between April 2008 and December 2010. Patients were assessed according to ESC 2006 guidelines for management of patients with stable angina and ACC/AHA 2005 guidelines for management of patients with unstable angina/non-STelevation myocardial infarction. The inclusion and exclusion criteria are shown in Table 1. Patients were free to withdraw from the study at any time. Potential participants who were interested in this study received a complete explanation of the protocol and signed the consent form. The ethical approval for the study was granted by the Ethics Committee of Guangzhou University of Traditional Chinese Medicine.

2.2. Design, Randomization, and Allocation. A double-blind randomized controlled clinical trial was conducted by using stratified randomized method. Center is a stratified factor in the present trial. Eligible participants after PCI were randomly assigned into 2 groups: treatment group who received conventional treatment and Huxin Formula or control group who received conventional treatment and the placebo. A sample size calculation based on the incidence rate of cardiovascular events in previous studies determined that 289 patients were needed to reduce the incidence rate of cardiovascular events of $8.5 \%$ (power 0.85 , significance level 0.05 ). With an estimated $15 \%$ dropout rate, we set the total sample size at 680 . Randomized codes were performed with SAS6.12 (statistical software package UNIFORM(n)). Eligible patients at each center were assigned randomization numbers from Institute of Basic Medical and Clinical Sciences, China Academy of Chinese Medical Sciences, which is responsible for the randomization of this research and was external to the trial. Participants and investigators were masked to group assignment. The prepared drugs were dispensed in similar looking bags, and the nature of the drug was concealed by the providers. The random allocation sequence was concealed until the data collection for the entire study was completed.

2.3. Treatment Protocols. Patients in the treatment group were provided Huxin Formula (in the form of granules) prepared by Jiangyin Pharmaceutical Co., Ltd. (Jiangsu, China). Every $10 \mathrm{~g}$ Huxin Formula granules consisted of ginseng (10 g), Exocarpium Citri Rubrum (5 g), Panax pseudoginseng $(8 \mathrm{~g})$, Pinellia ternata $(10 \mathrm{~g})$, Salvia miltiorrhiza $(10 \mathrm{~g})$, and Agastache $(10 \mathrm{~g})$ as crude drug. Patients in the control group were given placebo granules prepared by the same supplier. Placebo granules were designed to taste, smell, and look similar to Huxin Formula. Placebo consisted of amylum, bitter principle, excipient, and so forth. Huxin Formula or placebo was used once a day (10 $\mathrm{g}$ QD) after fully dissolving in $300 \mathrm{~mL}$ of boiled water cooled to $70^{\circ} \mathrm{C}$. Treatment started in one week after PCI and continued for 6 months (180 days). Then patients were followed up for another 6 months (180 days).

2.4. Outcomes. The baseline data, including gender, age, personal history, medical history, family history of cardiovascular disease, classification of cardiac function, and clinical classification of coronary heart disease, were assessed. Main cardiovascular events (death, nonfatal myocardial infarction, and repeat revascularization) were also observed and recorded. We assessed quality of life using two scales: (1) Seattle Angina Questionnaire (SAQ) and (2) scale of the life quality in integrative medicine for CAD (see Supplementary Material available online at http://dx.doi.org/10.1155/2014/ 143064). The outcomes were assessed at $0,90,180,270$, and 360 days after treatment.

2.5. Safety Monitoring. To assess the safety of the 6-month treatment, routine blood and liver and renal function tests were conducted before randomization, 90 days and 180 days after treatment. During the trial, adverse events were observed in detail and documented using case report forms.

2.6. Statistical Analysis. Statistical analysis sets included intention-to-treat (ITT) sets, per-protocol (PP) sets, and safety analysis sets. Descriptive analysis and inferential analysis of clinical features before and after treatment were as follows.

(1) Measurement data: functional indexes are presented as mean \pm standard deviation. Each group compared before and after treatment with paired $t$-test and Student's $t$-test was used to compare small sample data (including 95\% confidence interval). Rank-sum test (Wilcoxon test) is used for nonnormal distribution or heterogeneity of variance. Analysis of covariance or stratified analysis is used when baseline is not neat.

(2) Enumeration data: the constituent ratio and rate of each index were calculated. Two-group comparisons were performed using the fourfold table $\chi^{2}$ test (or accurate probability method) for the total effective rate and the $2 \times \mathrm{C}$ table $\chi^{2}$ test for the constituent ratio. 


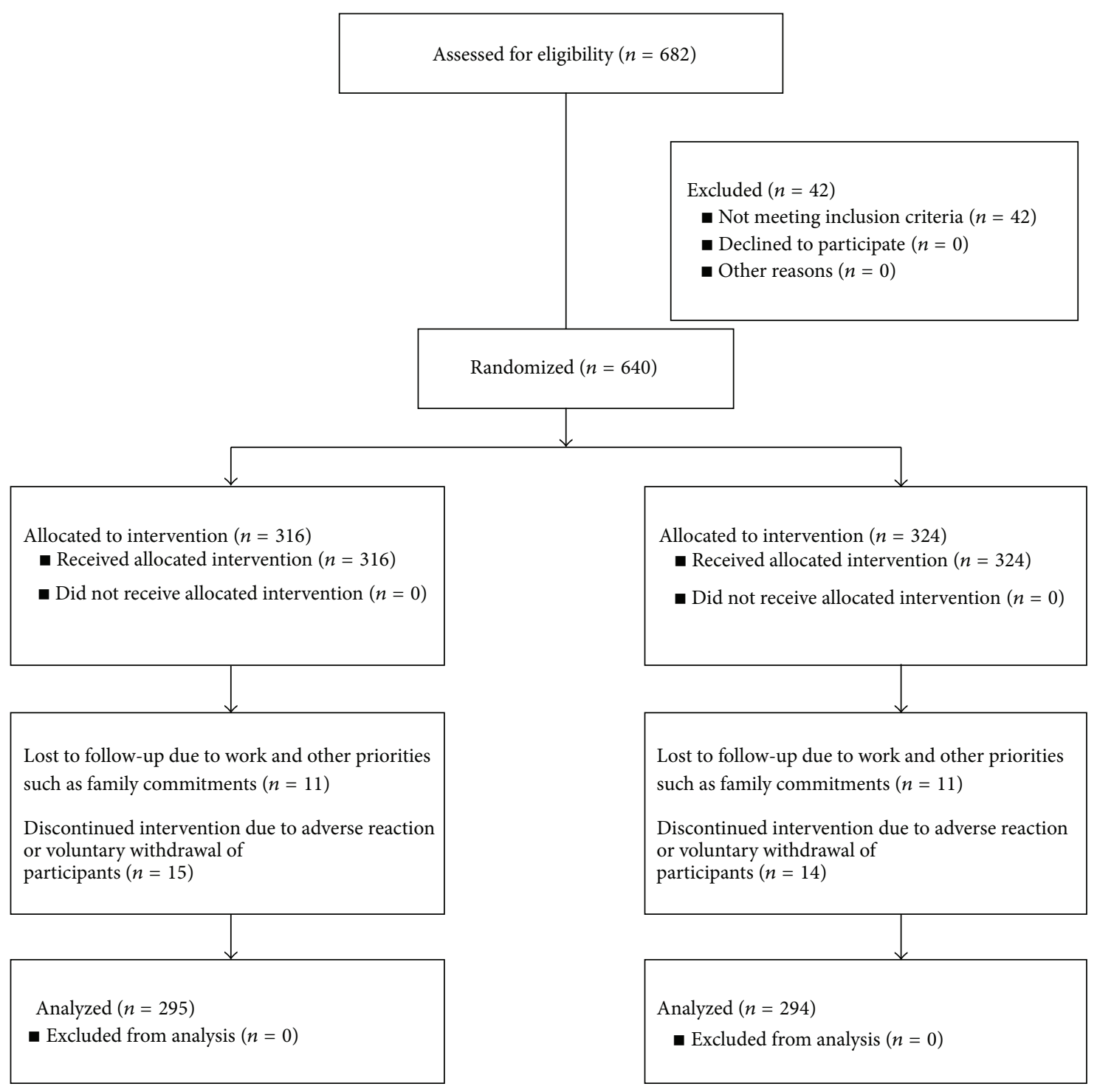

FIGURE 1: Consort 2010 flow diagram.

(3) Ranked data: two-group comparisons were performed using Mann-Whitney test.

(4) Life quality analysis: the general descriptive analysis is presented as mean \pm standard deviation, maximum, minimum, and median.

(5) The analysis of the factors affecting curative effect: the logistic regression analysis was used to analyze the factors that may affect the judgment of curative effect, including age, duration, classification of coronary heart disease, survival quality assessment scale of integrated traditional Chinese and western medicine, PCI operator, and center.

(6) The Kaplan-Meier method was used to compare the cardiovascular event rates and median time. Influence factors of cardiovascular events were analyzed by using the Cox regression analysis.

(7) Baseline comparison test level $\alpha=0.10$, and twogroup effects comparison test level $\alpha=0.05$.

Safety analysis of each case was mainly performed as descriptive statistics, including incidence and description of adverse events, the changes of laboratory testing results before and after test, and the relationship between abnormal changes and investigational drugs.

\section{Results}

3.1. Participants Flow. There were 682 patients with PCI treatment included in this research. 42 patients were eliminated, and 51 patients were withdrawn after 360 days. The flow of participants in the study is summarized in Figure 1. 
TABLE 2: Baseline clinical data $(n, \%)$.

\begin{tabular}{|c|c|c|c|}
\hline & Control group $(N=316)$ & Treatment group $(N=324)$ & $P$ \\
\hline Age $(\bar{x} \pm s)$ & $59.67 \pm 9.33$ & $60.91 \pm 8.97$ & 0.09 \\
\hline \multicolumn{4}{|l|}{ Gender } \\
\hline Male & $249(78.8)$ & $250(77.2)$ & \multirow{2}{*}{0.62} \\
\hline Female & $67(21.2)$ & $74(22.8)$ & \\
\hline Height $(\bar{x} \pm s)$ & $166.34+7.14$ & $166.04+7.37$ & 0.60 \\
\hline Weight $(\bar{x} \pm s)$ & $66.56 \pm 8.89$ & $64.82 \pm 9.53$ & 0.02 \\
\hline Pulse $(\bar{x} \pm s)$ & $74.6 \pm 11.93$ & $75.56 \pm 11.92$ & 0.31 \\
\hline Breath $(\bar{x} \pm s)$ & $19.52 \pm 2.03$ & $19.41 \pm 1.69$ & 0.46 \\
\hline Heart rate $(\bar{x} \pm s)$ & $74.71 \pm 12.01$ & $75.75 \pm 12.29$ & 0.28 \\
\hline $\operatorname{SBP}(\bar{x} \pm s)$ & $129.17 \pm 19.29$ & $130.30 \pm 22.00$ & 0.49 \\
\hline $\operatorname{DBP}(\bar{x} \pm s)$ & $77.21 \pm 11.44$ & $77.89 \pm 12.58$ & 0.47 \\
\hline \multicolumn{4}{|l|}{ Combined disease } \\
\hline Dyslipidemia & $96(30.4)$ & $91(28.1)$ & 0.52 \\
\hline Hypertension & $182(57.6)$ & $179(55.2)$ & 0.55 \\
\hline Diabetes & $72(22.8)$ & $60(18.5)$ & 0.18 \\
\hline Stroke & $16(5.1)$ & $13(4.0)$ & 0.52 \\
\hline Heart failure & $10(3.2)$ & $12(3.7)$ & 0.71 \\
\hline Gastrointestinal disorder & $31(9.8)$ & $26(8.0)$ & 0.43 \\
\hline Arrhythmia & $18(5.7)$ & $18(5.6)$ & 0.94 \\
\hline \multicolumn{4}{|l|}{ Medical history } \\
\hline Smoking & $159(50.3)$ & $165(50.9)$ & 0.88 \\
\hline Drinking & $50(15.8)$ & $52(16.0)$ & 0.94 \\
\hline Family history of CVD & $41(13.0)$ & $49(15.1)$ & 0.43 \\
\hline Allergy & $31(9.8)$ & $30(9.3)$ & 0.81 \\
\hline \multicolumn{4}{|l|}{ Clinical classification of CHD } \\
\hline Unstable angina & $168(53.2)$ & $193(59.6)$ & \multirow{2}{*}{0.10} \\
\hline $\mathrm{Ml}$ & $148(46.8)$ & $131(40.4)$ & \\
\hline \multicolumn{4}{|l|}{ Cardiac functions } \\
\hline $\mathrm{I}$ & $117(37.0)$ & $122(37.7)$ & \multirow{3}{*}{0.70} \\
\hline II & $157(49.7)$ & $166(51.2)$ & \\
\hline III & $42(13.3)$ & $36(11.1)$ & \\
\hline
\end{tabular}

CHD: coronary heart disease, CVD: cardiovascular disease, DBP: diastolic blood pressure, MI: myocardial infarction, and SBP: systolic blood pressure.

3.2. Baseline Data. The general characteristics of the patients are shown in Table 2. The comparisons between control group and treatment group were performed using the chisquare test for baseline classification data before treatment, including the demographic data (gender, ethnic, and cultural), the general situation (height, pulse, respiration, heart rate, systolic blood pressure, and diastolic blood pressure), personal history (history of smoking, drinking, and allergy and family history of cardiovascular disease), medical history (dyslipidemia, high blood pressure, diabetes, stroke, heart failure, and arrhythmia), classification of cardiac function, and clinical classification of coronary heart disease (unstable angina and myocardial infarction). There were no significant deviations $(P>0.05)$ between the two groups except the weight (66.56 versus 64.82).

3.3. Major Endpoints. There were no significant differences $(P>0.05)$ between control group and treatment group in major end points at 360-day follow-up (Table 3), including mortality, number of patients with nonfatal myocardial infarction, proportion of further revascularization, and rehospitalization caused by cardiovascular events.

3.4. Secondary Outcomes. There were no significant differences $(P>0.05)$ between control group and treatment group on secondary outcomes at all time points after PCI, including angina scale (frequency of attack, duration time, intensity of pain, and dose of nitroglycerin), SAQ (dimension of body activities limitation, angina pectoris stable state, heart attacks, disease knowledge, and treatment satisfaction), and the quality of life scale of integrative medicine.

\subsection{Stratified Analysis}

3.5.1. Stratified Analysis of EF before Treatment. As shown in Table 4, according to PCI per-protocol population and 
TABle 3: Major end points at 360 days.

\begin{tabular}{|c|c|c|c|c|c|}
\hline Events & & Control group $(n=295)$ & Treatment group $(n=294)$ & $\chi^{2}$ & $P$ value \\
\hline \multirow{2}{*}{ Death } & $\mathrm{Y}$ & $2(0.7)$ & $0(0.0)$ & \multirow{2}{*}{2.00} & \multirow{2}{*}{0.16} \\
\hline & $\mathrm{N}$ & $293(99.3)$ & $294(100.0)$ & & \\
\hline \multirow{2}{*}{ Nonfatal MI } & $\mathrm{Y}$ & $0(0.0)$ & $0(0.0)$ & \multirow{2}{*}{-} & \multirow{2}{*}{-} \\
\hline & $\mathrm{N}$ & $295(100.0)$ & $294(100.0)$ & & \\
\hline \multirow{2}{*}{ Repeat revascularization } & $\mathrm{Y}$ & $2(0.7)$ & $3(1.0)$ & \multirow{2}{*}{0.21} & \multirow{2}{*}{0.65} \\
\hline & $\mathrm{N}$ & $293(99.3)$ & $291(99.0)$ & & \\
\hline \multirow{2}{*}{ Readmission } & $\mathrm{Y}$ & $5(1.7)$ & $9(3.1)$ & \multirow{2}{*}{1.19} & \multirow{2}{*}{0.28} \\
\hline & $\mathrm{N}$ & $290(98.3)$ & $285(96.9)$ & & \\
\hline
\end{tabular}

Values are $n(\%)$. MI: myocardial infarction.

TABLE 4: Score of the quality of life scale for patients with EF $>50 \%(\bar{x} \pm s)$.

\begin{tabular}{|c|c|c|c|c|c|}
\hline Time & Group & $n$ & $\bar{x} \pm s$ & $Z$ & $P$ \\
\hline \multirow{2}{*}{0 day } & Control group & 244 & $71.80 \pm 12.62$ & \multirow{2}{*}{-0.24} & \multirow{2}{*}{0.81} \\
\hline & Treatment group & 251 & $72.03 \pm 12.18$ & & \\
\hline \multirow{2}{*}{90 days } & Control group & 244 & $81.32 \pm 9.592$ & \multirow{2}{*}{-0.42} & \multirow{2}{*}{0.68} \\
\hline & Treatment group & 251 & $81.79 \pm 9.220$ & & \\
\hline \multirow{2}{*}{180 days } & Control group & 244 & $82.84 \pm 9.24$ & \multirow{2}{*}{-0.67} & \multirow{2}{*}{0.51} \\
\hline & Treatment group & 251 & $83.39 \pm 8.58$ & & \\
\hline \multirow{2}{*}{270 days } & Control group & 244 & $84.72 \pm 8.77$ & \multirow{2}{*}{-0.66} & \multirow{2}{*}{0.51} \\
\hline & Treatment group & 251 & $85.26 \pm 8.431$ & & \\
\hline \multirow{2}{*}{360 days } & Control group & 244 & $86.29 \pm 8.55$ & \multirow{2}{*}{-1.04} & \multirow{2}{*}{0.30} \\
\hline & Treatment group & 251 & $87.11 \pm 7.70$ & & \\
\hline
\end{tabular}

stratified analysis of EF before treatment, for patients with $\mathrm{EF}>50 \%$, score of the quality of life scale was higher but not statistically significant $(P>0.05)$ in treatment group compared with control group at all time points.

\subsubsection{Stratified Analysis of the Type of Coronary Heart Disease} before Treatment. As shown in Table 5, according to PCI per-protocol population and stratified analysis of the type of coronary heart disease before treatment, for patients with unstable angina, score of the quality of life scale in 360 days was significantly higher in treatment group compared with control group $(P<0.05)$. There were no significant deviations $(P>0.05)$ between the two groups at another four time points.

3.6. Safety Analysis. Incidence of adverse event and specific event in treatment group was similar to control group. No obvious adverse reaction was found in the use of Huxin Formula.

\section{Discussion}

Coronary artery disease (CAD) is a major cause of mortality and morbidity in developed countries $[1,2]$. Before developing the technique of PCI, coronary artery bypass graft (CABG) had been the standard and the only revascularization procedure. With the development of medical imaging and heart catheterization techniques, the PCI, as an effective, safe, less disabling, and less expensive revascularization procedure, had become a more frequently used treatment than CABG for CAD in most western countries as well as in China. Growing evidence had showed that PCI can improve myocardial ischemia and reduce the risks of longterm adverse cardiovascular events $[3,4]$. The results of a meta-analysis of 13 randomized controlled trials showed that PCI with drug-eluting stents reduces the risks of major adverse cardiac events, recurrent myocardial infarction, and reintervention [5].

In recent years, other than biochemical endpoints, quality of life (QoL) is considered to be an important indicator of health outcome in CAD [6] and the most common indication for PCI [7]. Seattle Angina Questionnaire (SAQ) was the most commonly used instrument to capture comprehensive and sensitive changes in QoL of the cardiac patients [8-10]. Besides SAQ, scale of the life quality in integrative medicine for CAD, which was established for the first time, was used in the present research. The internal reliability of this scale was supported by the values of Cronbach's $\alpha$ that exceeded 0.7 for all the subscales. Scale of the life quality in integrative medicine for CAD was used to collect the mental and social functioning information that SAQ might have missed.

Although the role of PCI in chronic stable angina is well established as it alleviates ischemic symptoms and improves quality of life $[11,12]$, its benefit in general health status of patients with unstable angina and non-ST-elevation 
TABLE 5: Score of the quality of life scale for patients with unstable angina $(\bar{x} \pm s)$.

\begin{tabular}{|c|c|c|c|c|c|}
\hline Time & Group & $n$ & $\bar{x} \pm s$ & $Z$ & $P$ \\
\hline \multirow{2}{*}{0 day } & Control group & 168 & $69.61 \pm 11.27$ & \multirow{2}{*}{-0.32} & \multirow{2}{*}{0.75} \\
\hline & Treatment group & 193 & $69.97 \pm 10.65$ & & \\
\hline \multirow{2}{*}{90 days } & Control group & 168 & $80.75 \pm 8.94$ & \multirow{2}{*}{-0.53} & \multirow{2}{*}{0.59} \\
\hline & Treatment group & 193 & $81.09 \pm 8.86$ & & \\
\hline \multirow{2}{*}{180 days } & Control group & 168 & $82.44 \pm 9.03$ & \multirow{2}{*}{-1.06} & \multirow{2}{*}{0.29} \\
\hline & Treatment group & 193 & $83.33 \pm 8.11$ & & \\
\hline \multirow{2}{*}{270 days } & Control group & 168 & $84.78 \pm 8.18$ & \multirow{2}{*}{-0.72} & \multirow{2}{*}{0.47} \\
\hline & Treatment group & 193 & $85.23 \pm 8.25$ & & \\
\hline \multirow{2}{*}{360 days } & Control group & 168 & $85.70 \pm 8.08$ & \multirow{2}{*}{-2.51} & \multirow{2}{*}{$0.01^{\#}$} \\
\hline & Treatment group & 193 & $87.62 \pm 7.52$ & & \\
\hline
\end{tabular}

Significantly different versus control group $(P<0.05)$.

myocardial infarction (UA/NSTEMI) would not be sufficient. It is reported that poor QoL is highly prevalent in elderly patients undergoing PCI [13]. At 30 days after PCI, general health-related quality of life (HRQoL) was significantly lower $(0.86 \pm 0.21$ versus $0.89 \pm 0.17, P=0.001)$ after adjusting baseline characteristics $(P<0.001)$ [14]. Therefore, in addition to angina-specific therapy, comprehensive supportive care would be needed to improve the quality of life, which might improve long-term clinical outcome especially in patients after PCI.

According to the 2007 ACCF/AHA guidelines for the management of patients with unstable angina/non-STelevation myocardial infarction [15], clopidogrel is recommend to be used for at least one year. Lipid-lowering therapy with the use of statin has benefits in blocking the progression of coronary artery plaque and further preventing cardiovascular events and death [16]. However, effects of these treatments on relieving the symptoms and improving quality of life are not satisfying.

It is reported that some kinds of herbal medicine, such as Xuefu Zhuyu Capsule [17], exhibit better efficacy on HRQoL in patients with unstable angina after PCI. However, the application of TCM in the post-PCI patients still lacks support from rigorously designed clinical trial. Huxin Formula is formulated by modifying an experienced Chinese medicine formula which is created by a well-known Chinese doctor, Professor DENG Tie-tao, and has been widely used in clinical practice because of its marked effects on coronary heart disease $[18,19]$. Our earlier clinical study in 55 patients after CABG had showed that modified Huxin Formula can promote rehabilitation of the CABG patients, improving clinical symptoms and quality of life [20]. However, the effects of the formula in patients undergoing PCI were unknown. Therefore, the present study was designed to evaluate the efficacy and safety of Huxin Formula in patients after PCI.

Our results showed that, for patients with unstable angina, score of the quality of life scale in 360 days was significantly higher in treatment group $(87.62 \pm 7.52)$ compared with control group $(85.70 \pm 8.08)(P<0.05)$. This result suggested that Huxin Formula may improve the quality of life of patients after PCI compared to the placebo. There are 22 patients with the event of MACE (2 deaths, 6 repeat revascularizations, and 14 readmissions caused by cardiovascular events) in this research. The rate of one-year MACE event is only 3.23\%, slightly lower than 5-8\% that has been suggested in foreign related reports. There was no statistical difference between the two groups in other outcomes. Limited sample size and the relatively short observational period may be the two reasons for the negative results. Therefore, further studies with larger sample sizes and longer observational period are warranted on the base of these precious experiences.

\section{Conclusion}

In this research, the results showed that Huxin Formula is a safe treatment for patients after PCI. Though Huxin Formula failed to show superiority in primary and secondary outcomes, it has benefits in improving the quality of life in patients with unstable angina.

\section{Abbreviations}

$\begin{array}{ll}\text { ACS: } & \text { Acute coronary syndrome } \\ \text { BMS: } & \text { Bare metal stents } \\ \text { CABG: } & \text { Coronary artery bypass grafting } \\ \text { CAD: } & \text { Coronary artery disease } \\ \text { CHD: } & \text { Coronary heart disease } \\ \text { DES: } & \text { Drug-eluting stents } \\ \text { EF: } & \text { Ejection fraction } \\ \text { ITT: } & \text { Intention-to-treat } \\ \text { MACE: } & \text { Major adverse cardiovascular events } \\ \text { NSTEMI: } & \text { Non-ST-elevation myocardial infarction } \\ \text { PCI: } & \text { Percutaneous coronary intervention } \\ \text { PES: } & \text { Paclitaxel-eluting stents } \\ \text { PP: } & \text { Per-protocol } \\ \text { REVERSAL: Reversal of atherosclerosis with aggressive } \\ & \text { lipid lowering } \\ \text { SAQ: } & \text { Seattle Angina Questionnaire } \\ \text { SES: } & \text { Sirolimus-eluting stents } \\ \text { TCM: } & \text { Traditional Chinese medicine } \\ \text { UA: } & \text { Unstable angina. }\end{array}$




\section{Conflict of Interests}

The authors declare that there is no conflict of interests regarding the publication of this paper.

\section{Acknowledgments}

This work was supported by National Key Technology Research and Development Program of China (no. 2006BAI04A01) and registered in Clinical Trials.gov Protocol Registration system (ID NCT00965471). The authors thank Dr. Yingling Zhou from Guangdong Provincial People's Hospital, Dr. Dongqing An from Xinjiang Hospital of Chinese Medicine, Dr. Xiaohu Chen from Jiangsu Provincial Hospital of Chinese Medicine, Dr. Yawei Xu from The No. 10 Hospital of Shanghai, Dr. Zhengrong Deng from Shanxi Provincial People's Hospital, Dr. Li Li from Gansu Provincial People's Hospital, Dr. Zhimin Du from The First Affiliated Hospital of Sun Yat-sen University, Dr. Wei Wu from The First Affiliated Hospital of Guangzhou University of TCM, Dr. Zhicheng Li from The First Affiliated Hospital of Jinan University, Dr. Haiyu Yang from Jiangmen Wuyi Hospital of TCM, and Dr. Jinru Wei from People's Hospital of Liuzhou for participating in this trial.

\section{References}

[1] C. J. L. Murray and A. D. Lopez, "Global mortality, disability, and the contribution of risk factors: global burden of disease study," The Lancet, vol. 349, no. 9063, pp. 1436-1442, 1997.

[2] B. Brorsson, S. J. Bernstein, R. H. Brook, and L. Werkö, "Quality of life of chronic stable angina patients 4 years after coronary angioplasty or coronary artery bypass surgery," Journal of Internal Medicine, vol. 249, no. 1, pp. 47-57, 2001.

[3] R. Fattori and T. Piva, "Drug-eluting stents in vascular intervention," The Lancet, vol. 361, no. 9353, pp. 247-249, 2003.

[4] A. Marzocchi, F. Saia, G. Piovaccari et al., "Long-term safety and efficacy of drug-eluting stents: two-year results of the REAL (REgistro AngiopLastiche dell'Emilia Romagna) multicenter registry," Circulation, vol. 115, no. 25, pp. 3181-3188, 2007.

[5] P.-P. Hao, Y.-G. Chen, X.-L. Wang, and Y. Zhang, "Efficacy and safety of drug-eluting stents in patients with acute st-segmentelevation myocardial infarction: a Meta-Analysis of randomized controlled trials," Texas Heart Institute Journal, vol. 37, no. 5, pp. 516-524, 2010.

[6] W. Benzer, S. Höfer, and N. B. Oldridge, "Health-related quality of life in patients with coronary artery disease after different treatments for angina in routine clinical practice," Herz, vol. 28, no. 5, pp. 421-428, 2003.

[7] R. J. Gibbons, K. Chatterjee, J. Daley et al., "ACC/AHA/ACPASIM guidelines for the management of patients with chronic stable angina: a report of the American College of Cardiology/American Heart Association Task Force on Practice Guidelines (Committee on the Management of Patients with Chronic Stable Angina)," Journal of the American College of Cardiology, vol. 33, pp. 2092-2197, 1999.

[8] A. M. Borkon, G. F. Muehlebach, J. House, S. P. Marso, and J. A. Spertus, "A comparison of the recovery of health status after percutaneous coronary intervention and coronary artery bypass," Annals of Thoracic Surgery, vol. 74, no. 5, pp. 1526-1530, 2002.
[9] J. A. Spertus, A. C. Salisbury, P. G. Jones, D. G. Conaway, and R. C. Thompson, "Predictors of quality-of-life benefit after percutaneous coronary intervention," Circulation, vol. 110, no. 25, pp. 3789-3794, 2004.

[10] Z. Zhang, J. A. Spertus, E. M. Mahoney et al., “The impact of acute coronary syndrome on clinical, economic, and cardiacspecific health status after coronary artery bypass surgery versus stent-assisted percutaneous coronary intervention: 1Year results from the stent or surgery (SoS) trial," American Heart Journal, vol. 150, no. 1, pp. 175-181, 2005.

[11] W. S. Weintraub, J. A. Spertus, P. Kolm et al., "Effect of PCI on quality of life in patients with stable coronary disease," The New England Journal of Medicine, vol. 359, no. 7, pp. 677-687, 2008.

[12] M. T. D. Dyer, K. A. Goldsmith, L. S. Sharples, and M. J. Buxton, "A review of health utilities using the EQ-5D in studies of cardiovascular disease," Health and Quality of Life Outcomes, vol. 8, article 13, 2010.

[13] M. Singh, C. S. Rihal, R. J. Lennon, J. A. Spertus, K. S. Nair, and V. L. Roger, "Influence of frailty and health status on outcomes in patients with coronary disease undergoing percutaneous revascularization," Circulation: Cardiovascular Quality and Outcomes, vol. 4, no. 5, pp. 496-502, 2011.

[14] M.-J. Kim, D. S. Jeon, H.-C. Gwon et al., "Health-related qualityof-life after percutaneous coronary intervention in patients with UA/NSTEMI and STEMI: the Korean multicenter registry," Journal of Korean Medical Science, vol. 28, no. 6, pp. 848-854, 2013.

[15] J. L. Anderson, C. D. Adams, E. M. Antman et al., "ACC/AHA 2007 guidelines for the management of patients with unstable angina/non-ST-elevation myocardial infarction. A report of the American College of Cardiology/American Heart Association Task Force on Practice Guidelines (Writing committee to revise the 2002 guidelines for the management of patients with unstable angina/non-st-elevation myocardial infarction)," Journal of the American College of Cardiology, vol. 50, no. 7, pp. el-e157, 2007.

[16] S. E. Nissen, E. M. Tuzcu, P. Schoenhagen et al., "Effect of intensive compared with moderate lipid-lowering therapy on progression of coronary atherosclerosis: a randomized controlled trial," Journal of the American Medical Association, vol. 291, no. 9, pp. 1071-1080, 2004.

[17] F.-Y. Chu, J. Wang, K.-W. Yao, and Z.-Z. Li, "Effect of Xuefu Zhuyu Capsule on the symptoms and signs and health-related quality of life in the unstable angina patients with bloodstasis syndrome after percutaneous coronary intervention: a randomized controlled trial," Chinese Journal of Integrative Medicine, vol. 16, no. 5, pp. 399-405, 2010.

[18] W. Jiang, S. Li, W. Mao et al., "Effect of Huxin Formula on reverse cholesterol transport in ApoE-gene knockout mice," Chinese Journal of Integrative Medicine, vol. 18, no. 6, pp. 451456, 2012.

[19] X.-M. Ruan, W. Jiang, and Y. Lin, "Clinical efficacy of treatment for regulating $\mathrm{Pi}$ and protecting Xin in treating patients after coronary artery bypass grafting and its effect on patients' quality of life," Zhongguo Zhong Xi Yi Jie He Za Zhi, vol. 26, no. 1, pp. 28-32, 2006.

[20] X. M. Ruan, Y. Lin, W. Jiang, J. X. Hu, H. L. Wu, Q. X. Chen et al., "Effect of TCM method for regulating the spleen and protecting the heart on quality of life of patients undergoing coronary artery bypass grafting," Journal of Traditional Chinese Medicine, vol. 47, no. 8, pp. 587-590, 2006. 


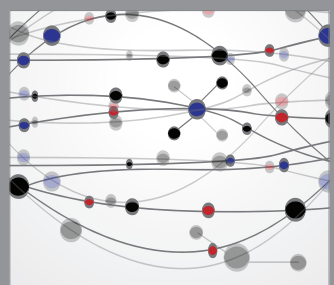

The Scientific World Journal
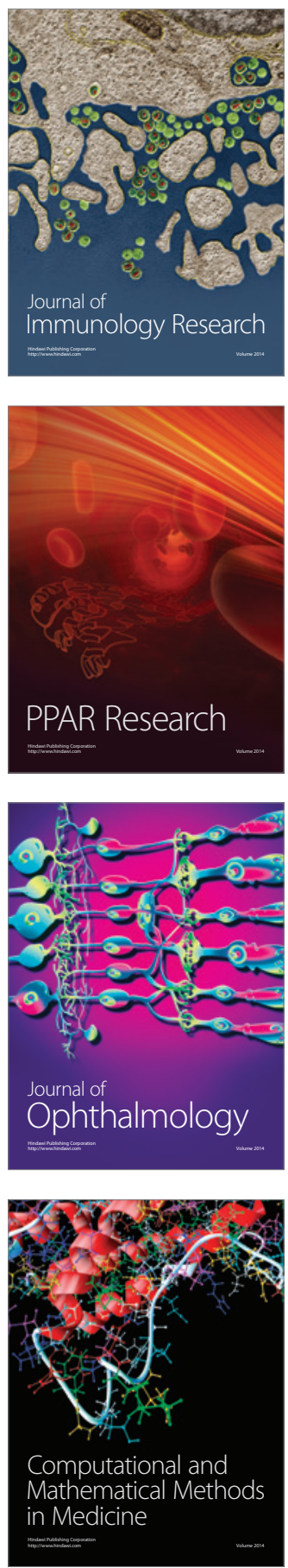

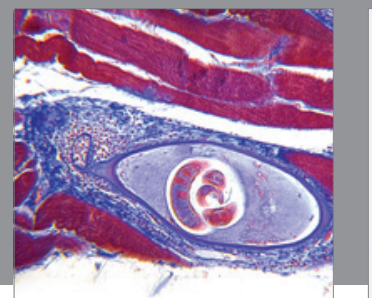

Gastroenterology

Research and Practice
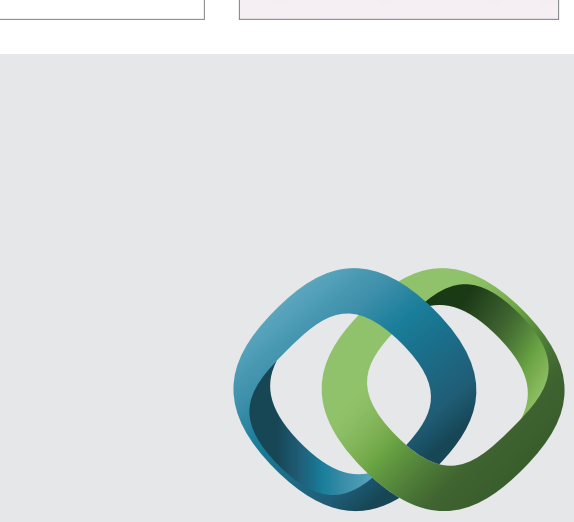

\section{Hindawi}

Submit your manuscripts at

http://www.hindawi.com
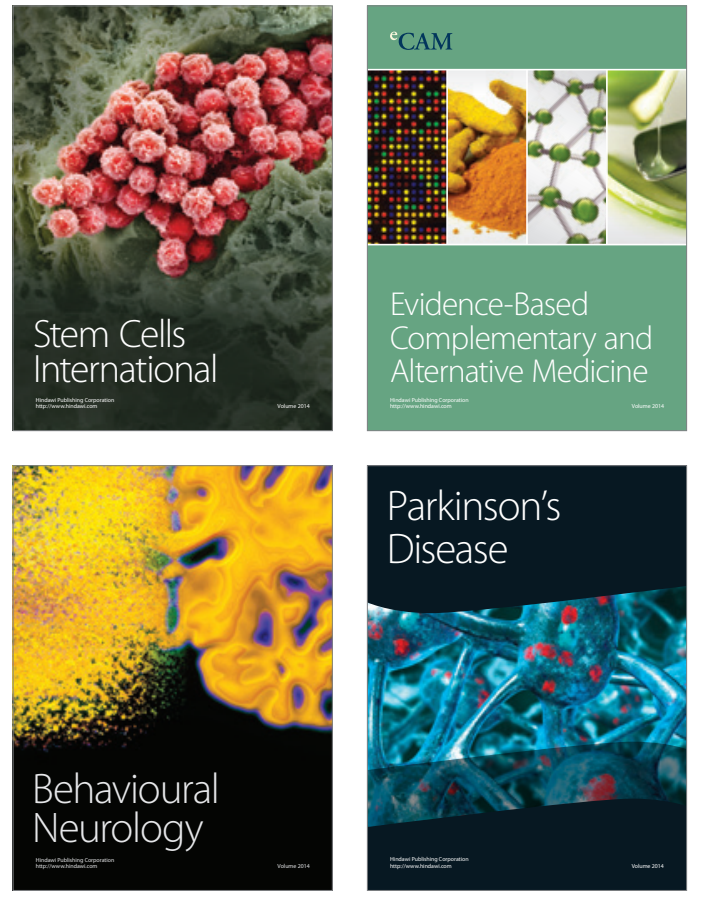
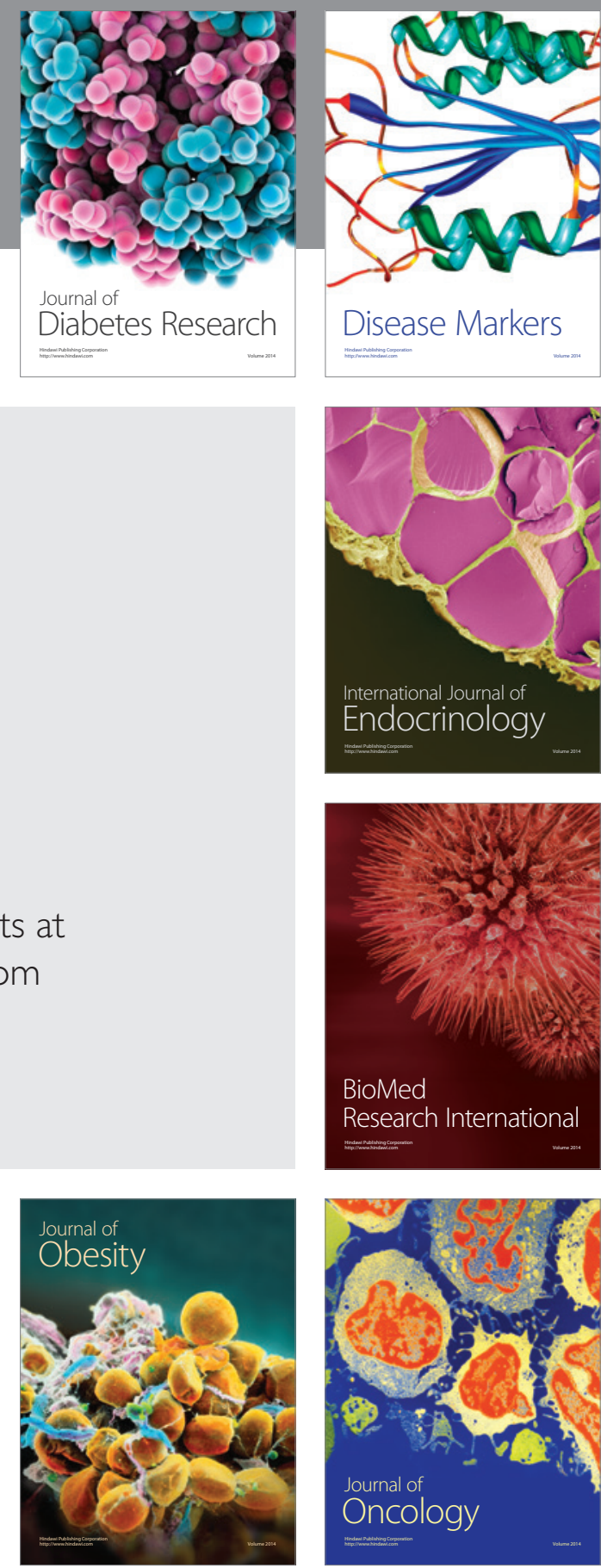

Disease Markers
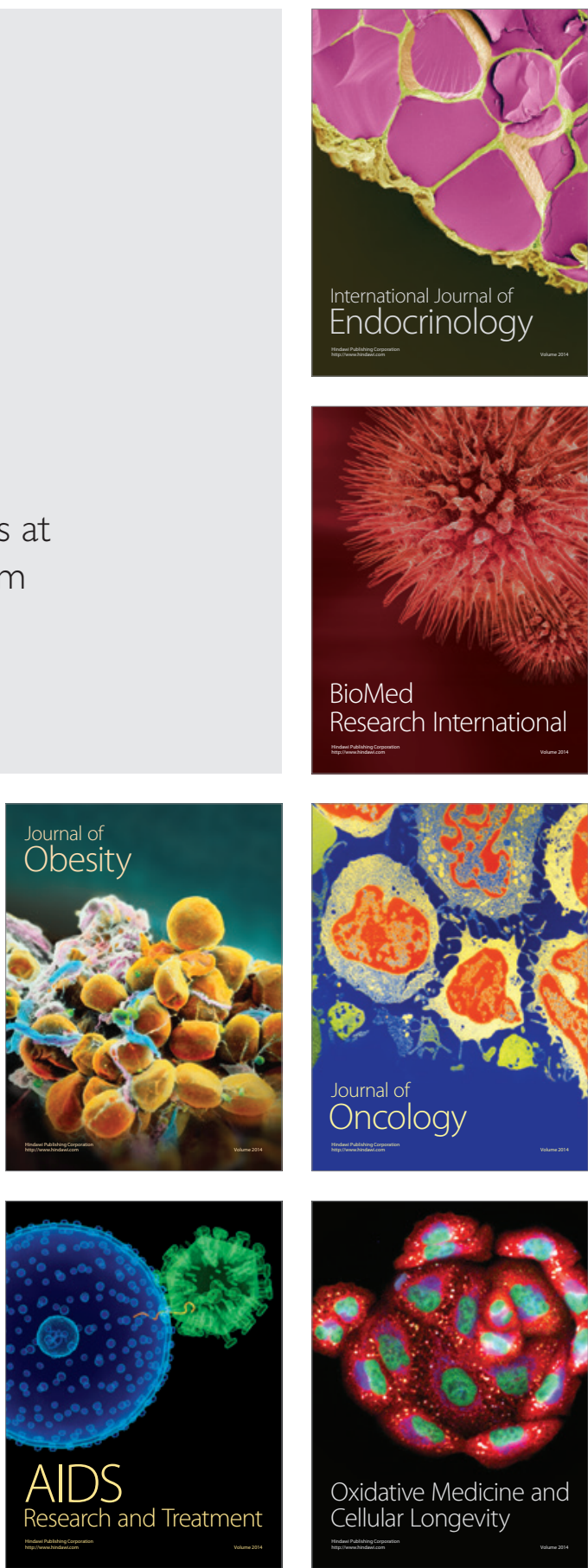